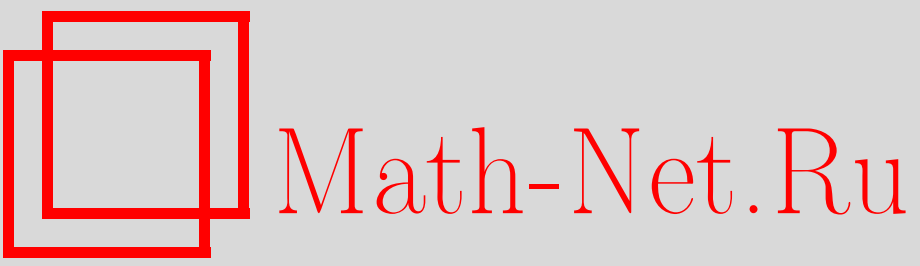

Ю. А. Кузнецов, Отсутствие арбитража в модели с фрактальным броуновским движением, УМН, 1999, том 54, выпуск 4, 175-176

DOI: https://doi.org/10.4213/rm191

Использование Общероссийского математического портала Math-Net.Ru подразумевает, что вы прочитали и согласны с пользовательским соглашением

http://www.mathnet.ru/rus/agreement

Параметры загрузки:

IP: 54.174 .149 .18

26 апреля 2023 г., 16:50:17 


\title{
ОТСУТСТВИЕ АРБИТРАЖА В МОДЕЛИ С ФРАКТАЛЬНЫМ БРОУНОВСКИМ ДВИЖЕНИЕМ
}

\author{
Ю. А. КУЗНЕцов
}

На фильтрованном вероятностном пространстве $\left(\Omega, \mathscr{F},(\mathscr{F} t)_{t \leqslant T}, \mathrm{P}\right)$ рассмотрим стандартное броуновское движение $B=\left(B_{t}\right)_{t \leqslant T}$ и фракталное броуновское движение $B^{H}=\left(B_{t}^{H}\right)_{t \leqslant T}$ с показателем $H, 0<H \leqslant 1$, т.е. гауссовский процесс с непрерывными траекториями и $B_{0}^{H}=0$, $\mathrm{E} B_{t}^{H}=0, \mathrm{E}\left(B_{t}^{H}\right)^{2}=t^{2 H}$,

$$
\operatorname{cov}\left(B_{t}^{H}, B_{s}^{H}\right)=\frac{1}{2}\left(|t|^{2 H}+|s|^{2 H}-|t-s|^{2 H}\right) .
$$

При $H=1 / 2$ фрактальное броуновское движение является броуновским движением. При $H=1$ имеем $\operatorname{Law}\left(B_{t}^{1} ; t \leqslant T\right)=\operatorname{Law}(\xi \cdot t ; t \leqslant T)$, где $\xi \sim \mathcal{N}(0,1)$.

Рассмотрим фрактальную версию модели Башелье $(B(r), S(\mu))$-рынка. Пусть для простоты $B_{t} \equiv 1(r=0), S_{t}=B_{t}^{H}(\mu=0)$.

При $H \in(0,1 / 2) \cup(1 / 2,1)$ процесс $B^{H}$ не является семимартингалом. Поэтому в этом случае не существует мартингальной меры, и первая фундаментальная теорема финансовой математики (отсутствие арбитража эквивалентно существованию мартингальной меры) не применима.

Пусть $\pi=(\beta, \gamma)$ - портфель с капиталом

$$
X_{t}^{\pi}=\beta_{t}+\gamma_{t} S_{t}, \quad t \leqslant T .
$$

Предположим, что $\pi$ - самофинансируемый, тогда

$$
X_{t}^{\pi}=X_{0}^{\pi}+\int_{0}^{t} \gamma_{u} d S_{u}
$$

В формуле (1) участвует интеграл по фрактальному броуновскому движению.

Определим этот интеграл согласно [1], [2].

ОПРЕДЕЛЕнИЕ. Стратегия $\pi$ доставляет арбитраж, если

$$
\left.X_{0}^{\pi}=0, \quad X_{T}^{\pi} \geqslant 0 \quad \text { (Р-п.н. }\right), \quad \mathrm{P}\left(X_{T}^{\pi}>0\right)>0 .
$$

Рассмотрим смешанную модель:

$$
S_{t}=\sigma_{H} B_{t}^{H}+\sigma B_{t} .
$$

Теорема. В смешанной модели арбитраж отсутствует в классе всех марковских стратегий $\gamma_{t}=G\left(S_{t}, t\right)$, где $G=G\left(S_{t}, t\right)$ класса $C^{1,1}$.

ДокАЗАТЕЛЬство. Не ограничивая общности, можно считать, что $\sigma_{H}=1, \sigma=1$. Тогда $S_{t}=B_{t}^{H}+B_{t}$. Пусть

По формуле Ито:

$$
\gamma_{t}=\frac{\partial F}{\partial x}\left(S_{t}, t\right)
$$

$$
d F\left(S_{t}, t\right)=\frac{\partial F}{\partial x}\left(S_{t}, t\right) d S_{t}+\left(\frac{\partial F}{\partial t}\left(S_{t}, t\right)+\frac{1}{2} \frac{\partial^{2} F}{\partial x^{2}}\left(S_{t}, t\right)\right) d t
$$
Капитал в момент времени $T$ равен $X_{T}^{\pi}=\int_{0}^{T} \frac{\partial F}{\partial x}\left(S_{t}, t\right) d S_{t}$. Условие неотрицательности $X_{T}^{\pi}$
записывается в виде

$$
F\left(S_{T}, T\right)-F(0,0) \geqslant \int_{0}^{T}\left(\frac{\partial F}{\partial t}\left(S_{t}, t\right)+\frac{1}{2} \frac{\partial^{2} F}{\partial x^{2}}\left(S_{t}, t\right)\right) d t .
$$


Можно считать, что $\Omega=\Omega_{1} \times \Omega_{2}$, и процесс $B_{t}^{H}$ определен на $\Omega_{1}$, а $B_{t}-$ на $\Omega_{2}$. Введем множества

$$
\begin{aligned}
A & =\left\{\omega=\left(\omega_{1} \times \omega_{2}\right): F\left(S_{T}, T\right)-F(0,0) \geqslant \int_{0}^{T}\left(\frac{\partial F}{\partial t}\left(S_{t}(\omega), t\right)+\frac{1}{2} \frac{\partial^{2} F}{\partial x^{2}}\left(S_{t}(\omega), t\right)\right) d t\right\}, \\
B= & \left\{\omega_{1}: F\left(S_{T}\left(\omega_{1} \times \omega_{2}\right), T\right)-F(0,0)\right. \\
& \left.\geqslant \int_{0}^{T}\left(\frac{\partial F}{\partial t}\left(S_{t}\left(\omega_{1} \times \omega_{2}\right), t\right)+\frac{1}{2} \frac{\partial^{2} F}{\partial x^{2}}\left(S_{t}\left(\omega_{1} \times \omega_{2}\right), t\right)\right) d t \mathrm{P}_{2} \text {-п.н. }\right\} .
\end{aligned}
$$

Имеем $\mathrm{P}(A)=1$. Тогда по теореме Фубини $\mathrm{P}_{1}(B)=1$.

Зафиксируем $\omega_{1} \in B$. Имеем $S_{t}\left(\omega_{1} \times \omega_{2}\right)=B_{t}^{H}\left(\omega_{1}\right)+B_{t}\left(\omega_{2}\right)$. Положим $C_{t}=B_{t}^{H}\left(\omega_{1}\right)$ при фиксированном $\omega_{1}$. Тогда неравенство

(3) $F\left(C_{T}+B_{T}\left(\omega_{2}\right), T\right)-F(0,0) \geqslant \int_{0}^{T}\left(\frac{\partial F}{\partial t}\left(C_{t}+B_{t}\left(\omega_{2}\right), t\right)+\frac{1}{2} \frac{\partial^{2} F}{\partial x^{2}}\left(C_{t}+B_{t}\left(\omega_{2}\right), t\right)\right) d t$

выполнено для почти всех $\omega_{2}$. Но это неравенство в точности означает неотрицательность капитала портфеля в обычной броуновской модели, когда $S_{t}=B_{t}\left(\omega_{2}\right)$ и стратегия $\gamma_{t}\left(\omega_{2}\right)=\frac{\partial F}{\partial x}\left(C_{t}+\right.$ $\left.B_{t}\left(\omega_{2}\right), t\right)$. Известно, что в этом случае арбитража нет [2], [3], и стратегия должна быть нулевой, T.e.

$$
\gamma_{t}\left(\omega_{2}\right)=\frac{\partial F}{\partial x}\left(C_{t}+B_{t}\left(\omega_{2}\right), t\right)=0
$$

Итак, при фиксированном $\omega_{1} \in B$ для почти всех $\omega_{2}$

$$
\frac{\partial F}{\partial x}\left(B_{t}^{H}\left(\omega_{1}\right)+B_{t}\left(\omega_{2}\right), t\right)=0
$$

для всех $0 \leqslant t \leqslant T$.

Отсюда ясно, что для всех $x$ и $t$

$$
\frac{\partial F}{\partial x}(x, t)=0 .
$$

Тем самым отсутствие арбитража в смешанной модели доказано.

\section{СПИСОК ЛИТЕРАТУРЫ}

[1] Lin S. J. // Stochastics Stochastics Rep. 1995. V. 55. № 1, 2. P. 121-140. [2] Ширяев А. Н. Основы стохастической финансовой математики. М.: Фазис, 1998. [3] Shiryaev A. N. On Arbitrage and Replication for Fractal Models. 